\title{
Population structure, genetic diversity and pathotypes of Streptococcus suis isolated during the last 13 years from diseased pigs in Switzerland
}

Simone Scherrer ${ }^{1 *} \mathbb{D}$, Giuliana Rosato ${ }^{2}$, Nathalie Spoerry Serrano ${ }^{1}$, Marc J. A. Stevens ${ }^{1}$, Fenja Rademacher ${ }^{1}$, Jacques Schrenzel ${ }^{3}$, Marcelo Gottschalk ${ }^{4}$, Roger Stephan ${ }^{1}$ and Sophie Peterhans ${ }^{1}$

\begin{abstract}
Streptococcus (S.) suis is a globally important swine pathogen, which comprises certain zoonotic serotypes. In this study, a detailed characterization of 88 porcine $S$. suis isolates was performed by analyzing capsular (cps) types, multilocus sequence typing (MLST) and investigation of the minimum core genome (MCG). In order to focus on the virulence potential of presumable invasive disease-associated S. suis isolates, virulence-associated gene profiles were assessed followed by screening a chosen subset of S. suis strains with a molecular pathotyping tool. Results showed a high genetic variability within this strain collection. In total, seventeen cps types were identified with a predominance of cps type 9 (15.9\%) and 6 (14.8\%). MLST revealed 48 sequence types (STs) including 41 novel ones. The population structure of S. suis was heterogenous and isolates belonged to eight different clonal complexes (CCs) including CC28 (9.1\%), CC1109 (8\%), CC13/149 (6.8\%), CC1237 (5.7\%), CC1 (3.4\%), CC17 (3.4\%), CC87 (2.3\%), and CC1112 (1.1\%), whereas a significant portion of isolates (60.2\%) could not be assigned to any described CCs. Virulence-associated markers, namely extracellular protein factor (epf), muramidase-released protein (mrp), and suilysin (sly), showed a link with STs rather than with cps types. With this study an expanded knowledge about the population structure and the genetic diversity of $S$. suis could be achieved, which helps to contribute to an optimal public health surveillance system by promoting a focus on strains with an increased virulence and zoonotic potential.
\end{abstract}

Keywords: Streptococcus suis, MLST, Capsular type, Clonal complex, Virulence potential, Invasive disease-associated, MCG groups

\section{Introduction}

Streptococcus suis (S. suis) is a Gram-positive bacterium recognized to be an important swine pathogen responsible for various diseases including septicemia with sudden death, meningitis, endocarditits, polyserositis, and arthritis [1]. S. suis also colonizes the upper respiratory tract with commensal strains giving rise to carrier pigs [2].

*Correspondence: simone.scherrer@uzh.ch

${ }^{1}$ Department of Veterinary Bacteriology, Institute for Food Safety and Hygiene, Vetsuisse Faculty, University of Zurich, Zurich, Switzerland

Full list of author information is available at the end of the article
Moreover, S. suis can appear as an opportunistic pathogen in combination with other pathogens [3]. In addition, infection with $S$. suis not only leads to major economic problems due to important losses in pig production worldwide, but the bacterium is also capable of infecting humans. In Western countries, infection of humans usually involves a single person such as pig farmers, veterinarians, people who work at abattoir, or butchers. Infection mainly takes place through direct contact of skin wounds with contaminated pork even in cases where no obvious wound is present [4]. On the other hand, in 
Southeast Asian countries, the main route of infection seems to be the gastrointestinal tract where consumption of undercooked pork products or a traditional soup containing raw pig blood is common and humans frequently live in close contact to pigs [5]. The most frequently described manifestations in humans are meningitis and septic shock [6].

Currently, there are at least 29 S. suis serotypes [7] described based on a serological reaction against the capsular polysaccharide, which has been commonly reported to be a major virulence factor with antiphagocytic properties [8]. Strains of heterogeneous serotype 2, which are responsible for more than $80 \%$ of human cases, represent a globally emerging zoonotic threat [9]. To a lesser extent, serotype 14 is also associated with disease in both humans and pigs [10]. Worldwide, major S. suis serotypes isolated from diseased pigs are serotypes 2, 9, 3, 1/2 and 7 [9]. In some European countries, serotype 9 strains are emerging and are considered to be responsible for causing disease among pigs [11] and sporadically capable to infect humans as reported in one case report from Thailand [12].

The most commonly described virulence markers for S. suis include muramidase-released protein (MRP) [13], extracellular protein factor (EF) [14], and the hemolysin suilysin (SLY) [15], which were mainly associated with a virulence potential of $S$. suis serotype 2 strains [16]. MRP is a surface protein anchored to the cell wall [17] and has recently been shown to be a major fibrinogen-binding protein [18]. Human fibrinogen bound to MRP increases viability of S. suis serotype 2 in human blood and its capability of migrating across the human cerebral endothelial cell barrier, thereby promoting the development of meningitis [19]. EF is secreted independent of an interaction between bacteria and host cell involved in enhancing bacterial infection, invasion, and pathogenicity [20]. Both MRP and EF have variants of different molecular weights $[21,22]$. Strains harboring the short form of epf are considered the most virulent form of the epf subtypes [21]. SLY is a thiol-activated toxin antigenically related to cholesterol-binding toxins, which forms transmembrane pores [23] and is described to be toxic to phagocytic, endothelial, and epithelial cells [24]. However, since isogenic mutants can be found, which do not express MRP, EFP, and/or SLY and are still virulent, it is more precise to term those factors as virulence-associated markers [14].

Multilocus sequence typing (MLST) is a useful method to examine the population structure and provide epidemiological tracing of pathogenic bacteria. It has been applied to various species and is a valuable tool that allows further investigation of the global distribution and evolution of several pathogens [25], including S. suis as reported in former studies $[26,27]$. The classification of sequence types (STs) resulting from MLST allows grouping of genetically similar genotypes in clonal complexes (CCs), thus revealing the population structure of S. suis. Various CCs are identified globally, whereas the prevalence of certain genotypes can vary in different geographical regions. The most important CCs implicated in human infections are the following: CC1 (harboring most important ST1) in Europe, Asia, North- and South America, and Africa [28]; CC20 in the Netherlands [11]; CC25 and CC28 in North America, Australia, and Asia [28], although in the United States an increasing trend of the genetically diverse CC94 was observed [29]. In Europe, a high prevalence of virulent $m r p+e p f+s l y+c p s 2$ strains being part of $\mathrm{CC} 1$ and $m r p^{*} c p s 9$ strains of $\mathrm{CC} 16 / 87$ can be noticed [30].

A molecular surveillance tool to predict the virulence potential of $S$. suis, by differentiating potentially invasive disease-associated strains from commensal or possibly opportunistic non-disease-associated strains is of advantage. Such a pathotyping tool was recently described by Wileman et al. [31] and, provided that its application is successful, could be used as a convenient and promising diagnostic assay enabling the detection of highly virulent emerging strains. Based on whole genome sequencing data, three pathotyping markers were identified [31]. First of all, a disease-associated marker was described, which is predicted to be a copper-exporting ATPase (SSU207) potentially playing an essential role in copper homeostasis in Gram-positive and -negative bacterial pathogens $[31,32]$. The second proposed disease-associated marker is annotated as type I restriction-modification (RM) system S protein (SSU1589) in S. suis strain P1/7 (GenBank accession number NC_012925.1) and is supposed to be implicated in the defense of host bacteria [33]. The third genetic marker is thought to be non-disease-associated and the corresponding protein is suggested to be a putative sugar ATP-binding cassette $(\mathrm{ABC})$ transporter (SSUST30534).

The aim of this study was to determine the genetic diversity of Swiss porcine $S$. suis isolates collected during the past 13 years and to assess their virulence potential. Observed phenotypes arising from pathological, histological and bacteriological examinations were compared to the virulence-associated genotype profiles obtained by analyzing the classical virulence-associated markers ( $m r p$, efp and $s l y$ ) and by classifying the S. suis population using a system based on minimum core genome (MCG). Moreover, a selected subset of S. suis strains, based on a comprehensive data availability of histopathological findings of the corresponding clinical cases, was screened with an available novel pathotyping tool [31]. The identification of major pathogenic strains could help to establish an optimal public health surveillance system 
by promoting a focus on strains with an increased virulence and zoonotic potential.

\section{Materials and methods Streptococcus suis isolates}

In this study a total of 88 porcine isolates obtained from routine diagnostic submissions to the Department of Veterinary Bacteriology at the Vetsuisse Faculty, University of Zurich, within the time span of 2006-2019, were included. The samples were recovered from 77 independent farms located in three main regions of Switzerland (Central- and Eastern part, and Canton of Zurich) with one to three samples per farm always derived from different animals. No isolates came from the French speaking Western part of Switzerland and the Canton of Bern. Isolates of the same farm were included only if STs were different. Samples analyzed originated from the following sites: $34.1 \%(n=30 / 88)$ from blood, $20.5 \%$ $(n=18 / 88)$ from brain, $14.8 \%(n=13 / 88)$ from lung, $11.4 \%(n=10 / 88)$ from joint, $8 \%(n=7 / 88)$ from heart, $3.4 \%(n=3 / 88)$ from genital tract, $2.3 \%(n=2 / 88)$ each from abscess and kidney, and $1.1 \%(n=1 / 88)$ each from abdominal cavity, nasal swab and liver (Table 1). In order to verify the relevance of the $S$. suis isolates, the results of the histological examination were consulted. S. suis infection was considered as relevant in cases where the histological diagnoses included meningitis, polyarthritis, endocarditis, or placentitis, and when histological features consistent with septicemia [34] were observed. In two cases no histopathology was performed, but $S$. suis was determined relevant due to clear bacteriological findings (pure culture of S. suis), appropriate anamnesis and clinical signs. Isolates, for which a clear link to $S$. suis could be ruled out histopathologically (due to

\begin{tabular}{lc}
$\begin{array}{l}\text { Table } 1 \text { Number of porcine } \\
\text { corresponding site of isolation. }\end{array}$ & suis isolates from \\
\hline Site of isolation & $\begin{array}{l}\text { Number } \\
\text { of isolates }\end{array}$ \\
\hline Blood & 30 \\
Brain & 18 \\
Lung & 13 \\
Joint & 10 \\
Heart & 7 \\
Genital tract & 3 \\
Abscess & 2 \\
Kidney & 2 \\
Abdominal cavity & 1 \\
Nasal swab & 1 \\
Liver & 1 \\
Total & 88 \\
\hline
\end{tabular}

inappropriate age or due to another etiological diagnosis), were termed as not relevant. Isolates with an incomplete record of data, no S. suis relevance could be assigned and were therefore excluded for screening with the pathotyping tool.

\section{DNA extraction and species identification}

Isolates were cultured for 24 to $48 \mathrm{~h}$ at $37^{\circ} \mathrm{C}$ on Columbia blood sheep agar plates (Thermo Fisher Diagnostics AG, Pratteln, Switzerland) under aerobic conditions. DNA was extracted using a standard heat lysis protocol [35]. Briefly, colony material was dissolved in lysis buffer containing Proteinase $\mathrm{K}$ at $60{ }^{\circ} \mathrm{C}$ for 45 min under constant shaking followed by a heat deactivation step at $96{ }^{\circ} \mathrm{C}$ for $15 \mathrm{~min}$. DNA samples were diluted to $20 \mathrm{ng} / \mu \mathrm{l}$ for subsequent reactions.

The isolates were identified by matrix-assisted laser desorption ionization-time-of-flight mass spectrometry (MALDI-TOF MS, Bruker, Bremen, Germany). In addition, to confirm S. suis species, a recN (recombination/ repair protein gene) PCR was performed as described earlier [36].

\section{Capsular typing and MLST}

Capsular typing was performed as previously described [37]. Universal primers targeting 16S rRNA gene were used for internal control of the PCR reactions [38]. PCR products were analyzed on a capillary electrophoresis QIAxcel Advanced device (Qiagen, Hilden, Germany) using a screening cartridge, QX $15 \mathrm{bp}-3 \mathrm{~kb}$ alignment marker and QX $100 \mathrm{bp}-2.5 \mathrm{~kb}$ size marker (Qiagen) according to the manufacturer's instructions. The resulting electropherograms were viewed with the QIAxcel ScreenGel 1.2.0 software (Qiagen). Isolates with capsular (cps) type pair 2 or $1 / 2$ and 1 or 14 were assigned to the corresponding cps type using a recently developed HRM assay [39].

MLST was performed targeting aroA, cpn60, dpr, gki, recA, thrA and mutS as described previously [26]. For strains with no PCR amplification using primer mutS, alternative mutS primers have been used as described [40]. Briefly, PCR was set up in a final volume of reaction mixture of $20 \mu \mathrm{l}$ containing HotStartTaq Master Mix (Qiagen), $0.5 \mu \mathrm{M}$ of each primer pair and $20 \mathrm{ng}$ template DNA with cycling conditions as described [26]. Sequencing of the seven housekeeping genes was performed by Sanger sequencing (Microsynth, Balgach, Switzerland). CLC Main Workbench 7.0.1 was used for sequence analysis. STs were assigned based on PubMLST database (https://pubmlst.org/ssuis). New housekeeping gene alleles were submitted to the database in order to create new STs. For visualizing groups of related genotypes and for identification of CCs, Swiss isolates were clustered at 
triple locus variant level with all isolates from the $S$. suis database (2289 isolates on September 27, 2019). The goeBURST algorithm from Phyloviz software [41] was used to visualize CCs and to create a MLST-based minimal spanning tree. CCs comprised STs with at least six identical alleles. STs that did not form any group were defined as singletons and unrelated STs grouping only with individual STs were indicated as non-defined founders.

\section{Virulence-associated gene profiling and MCG typing}

All $S$. suis isolates were tested for three virulence-associated genes ( $m r p$, epf, sly) by multiplex PCR using primers as described previously [22]. Additionally, a epf monoplex PCR [22] was conducted to ensure reliable detection of large variants of the epf gene designated as epf $f^{*}$ [21]. To accurately differentiate size variants of $m r p$, a PCR was performed using the $m r p$ variant primers as described previously [22]. The PCR assays were performed using HotStartTaq Master Mix (Qiagen) according to the manufacturer's instructions. MCG sequence typing was performed as described previously [42].

\section{Pathotyping}

Pathotyping was carried out using a recently developed multiplex PCR [31] by analyzing a selected subset of 75 isolates, which could be linked without any doubt to either etiologically relevant $S$. suis diseased or not $S$. suis relevant diseased pigs (Table 2). Briefly, a multiplex PCR was conducted using primers targeting two diseaseassociated genes (copper exporting ATPase 1 and type I RM system $\mathrm{S}$ protein), one non-disease-associated marker (putative sugar ABC transporter), and S. suis specific primers (sporulation regulator WhiA). The multiplex PCR was executed using HotStartTaq Mix (Qiagen) applying the three-step thermal cycling program as described previously [31]. PCR products were analyzed on a capillary electrophoresis QIAxcel Advanced device (Qiagen) using a screening cartridge, QX $15 \mathrm{bp}-3 \mathrm{~kb}$ alignment marker and QX $100 \mathrm{bp}-2.5 \mathrm{~kb}$ size marker (Qiagen) according to the manufacturer's instructions.

\section{Whole genome sequencing of a selected panel of isolates}

Whole genome sequencing (WGS) of a selected panel comprising 13 Swiss clinical S. suis strains of porcine origin has been performed previously [43]. Strains were chosen based on the aim to cover isolates of the most prevalent cps types (cps6 and cps9) and cps types most likely supposed to be virulent (cps $1 / 2$, cps 1 and $c p s 2)$ taking into consideration different years of sampling. Briefly, genomic DNA was isolated with the DNA Blood and Tissue Kit (Qiagen) and sequencing was performed on a MiniSeq sequencer (Illumina, San Diego, USA) with 150-bp paired-end reads using the Nextera DNA Flex kit
(Illumina). Sequences are available under GenBank SRA accession numbers SRR8290472-SRR8290486.

\section{Results \\ Pathological and bacteriological findings}

In total, 88 isolates were obtained. Detailed histopathological investigations and bacteriological examinations revealed 52 isolates as $S$. suis relevant and 23 isolates as not $S$. suis relevant. For 13 isolates no detailed records were available and therefore, were not included in the comparison with the pathotyping tool. The most frequently reported history was found to be occurrence of sudden death of one or more pigs on a farm $(n=29 / 88)$ and observations of neurological symptoms $(n=24 / 88)$. In terms of post-mortem findings, the most commonly identified reason of death found by pathologists was septicemia $(n=27 / 75)$ and meningitis $(n=27 / 75)$.

\section{Characterization of S. suis isolates by cps typing and MLST} Cps typing of the isolates identified a distribution of seventeen different cps types comprising $89.8 \%$ of isolates, whereas in $10.2 \%$ of cases no cps type could be assigned and were therefore classified as nontypeable (NT) (Additional file 1). Capsular gene analysis yielded two main cps types: $15.9 \%$ cps $9(n=14)$ and $14.8 \%$ cps $6(n=13)$. Furthermore, the following cps types were identified: $c p s 1$ $(n=6), \operatorname{cps} 15(n=6)$, cps $16(n=6)$, cps $7(n=4)$, cps 2 $(n=4)$, cps $8(n=3)$, cps $3(n=4)$, cps $1 / 2(n=3)$, cps 12 $(n=3)$, cps4 $(n=3)$, cps31 $(n=3), \operatorname{cps} 5(n=2), \operatorname{cps} 21$ $(n=2), \operatorname{cps} 28(n=2), \operatorname{cps} 19(n=1)$, and NT $(n=9)$. Clinically observed $S$. suis relevant phenotypes confirmed by histopathological analysis was detected for isolates of cps 1, cps 2 , cps6, and cps7. In contrast, non-relevant $S$. suis features could explicitly be associated with isolates of cps8.

MLST analysis revealed that $22.7 \%$ of isolates belonged to eight previously identified STs, while $77.3 \%$ had a new MLST profile encompassing 41 new STs. The predominant STs were ST1104 (cps6, $n=13 / 13$ ) comprising $14.8 \%$ of isolates, followed by $9.1 \%$ of ST1105 (cps9, $n=8 / 14)$, and $6.8 \%$ of isolates comprised ST13 (cps1, $n=6 / 6)$. The newly assigned STs in this study were ST1099-ST1130, ST1133, ST1134, ST1237-ST1240, ST1243, and ST1244 (Additional file 2). Three isolates of cps $2(n=3 / 4)$ were an atypical single locus variant (SLV) of ST1 (ST1103), whereas one cps 2 isolate $(n=1 / 4)$ was identified as a relatively common ST28 (Figure 1). The distribution of STs in relation to cps type highlights the diversity of $S$. suis. Thirteen of the seventeen cps types identified enclosed multiple STs, ranging from two to six different STs per cps type. Cps1, cps4, cps6, and cps 19 only harbored one $\mathrm{ST}$, whereas cps 16 showed the greatest variety of six different STs (Figure 1). 
Distribution of STs in relation to year of isolation

Figure 2 illustrates a MLST-based minimal spanning tree of all tested isolates within a time span of 2006-2019. ST1104 (cps6, $n=13 / 13$ ) and ST1105 (cps9, $n=8 / 14$ ) were the most prevalent STs identified in respect to the temporal distribution recovered during eight and six different years, respectively. ST13 (cps1, $n=6 / 6$ ) was collected in four different years. ST17 (cps4, $n=3 / 3$ ), ST29 (cps7, $n=3 / 4)$, ST231 (cps9, $n=1 / 14 ;$ cps $3, n=2 / 4)$, ST1103 (cps2, $n=3 / 4$ ), and ST1236 (cps9, $n=3 / 14$ ) originated from three different years. ST1101 (cps15, $n=2 / 6)$ and ST1109 (cps8, $n=1 / 3$; cps $9, n=1 / 14)$ were found

Table 275 porcine S. suis isolates evaluated with the pathotyping tool.

\begin{tabular}{|c|c|c|c|c|c|c|c|c|c|c|c|c|c|c|}
\hline Strain ID & $\begin{array}{l}\text { histopathological } \\
\text { findings }\end{array}$ & $\begin{array}{c}\text { S. suis } \\
\text { relevance }\end{array}$ & $\begin{array}{l}\text { cps } \\
\text { type }\end{array}$ & epf & $m r p$ & sly & MLST & $\begin{array}{c}\text { Clonal } \\
\text { Complex }\end{array}$ & MCG & SSU0577 & SSU1589f & SSU0207f & SSUST3_0534 & Pathotpying result \\
\hline PP 423 & septicemia & relevant & $1 / 2$ & - & $m r p^{* * *}$ & - & 1133 & CC28 & 4 & + & - & $+^{*}$ & - & disease-associated \\
\hline MB 1192 & pneumonia & not relevant & $1 / 2$ & - & $m r p^{s}$ & - & 28 & CC28 & 4 & + & - & $+^{*}$ & - & disease-associated \\
\hline MB 1598 & meningitis & relevant & 1 & - & - & sly & 13 & CC13/149 & $\mathrm{N}$ & + & - & - & - & non-disease-associated \\
\hline PP 269 & septicemia & relevant & 1 & - & - & sly & 13 & CC13/149 & $\mathrm{N}$ & + & - & - & - & non-disease-associated \\
\hline PP 735 & polyarthritis & relevant & 1 & - & - & sly & 13 & $\mathrm{CC} 13 / 149$ & $\mathrm{~N}$ & + & - & - & - & non-disease-associated \\
\hline PP 730 & meningitis, polyarthritis & relevant & 1 & - & - & sly & 13 & CC13/149 & $\mathrm{N}$ & + & - & - & - & non-disease-associated \\
\hline MB 1635 & polyarthritis, septicemia & relevant & 1 & - & - & sly & 13 & CC13/149 & $\mathrm{N}$ & + & - & - & - & non-disease-associated \\
\hline MB 1656 & meningitis, polyarthritis & relevant & 1 & - & - & sly & 13 & CC13/149 & $\mathrm{N}$ & + & - & - & - & non-disease-associated \\
\hline MB 1329 & meningitis & relevant & 2 & - & $m r p$ & sly & 1103 & CC1 & 1 & + & + & + & - & disease-associated \\
\hline MB 1555 & * & relevant & 2 & - & $m r p$ & sly & 1103 & CC1 & 1 & + & + & + & - & disease-associated \\
\hline $19-1715-1$ & * & relevant & 2 & $e p f^{*}$ & $m r p$ & sly & 1103 & $\mathrm{CC} 1$ & 1 & + & + & + & - & disease-associated \\
\hline PP 463 & endocarditis valvularis & relevant & 2 & - & $m r p^{5}$ & - & 28 & CC28 & 4 & + & - & $+*$ & - & disease-associated \\
\hline MB 1313 & meningitis & relevant & 3 & - & - & sly & 231 & CC1109 & $\mathrm{N}$ & + & - & $+^{*}$ & - & disease-associated \\
\hline MB 454 & septicemia & relevant & 3 & - & - & sly & 1113 & CC1109 & $\mathrm{N}$ & + & - & $+^{*}$ & - & disease-associated \\
\hline MB 1355 & peritonitis, polyarthritis & not relevant & 3 & - & $m r p^{* * *}$ & - & 856 & $\mathrm{CC} 28$ & 4 & + & - & $+*$ & - & disease-associated \\
\hline PP 414 & meningitis & relevant & 4 & epf & $m r p^{\mathrm{s}}$ & sly & 17 & CC17 & 1 & + & - & + & - & disease-associated \\
\hline PP 772 & pneumonia & not relevant & 4 & $e p f$ & $m r p^{\mathrm{s}}$ & sly & 17 & CC17 & 1 & + & - & + & - & disease-associated \\
\hline MB 906 & meningitis, peritonitis & not relevant & 5 & - & - & - & 1114 & $\mathrm{~S}^{\mathrm{c}}$ & ungroupable $^{d}$ & + & - & - & + & non-disease-associated \\
\hline MB 1158 & endocarditis valvularis & relevant & 5 & - & - & - & 1124 & $\mathrm{Sc}^{\mathrm{c}}$ & novel1 ${ }^{\mathrm{e}}$ & + & - & - & - & non-disease-associated \\
\hline PP 425 & meningitis & relevant & 6 & - & - & - & 1104 & ndfc & ungroupable $^{d}$ & + & - & - & - & non-disease-associated \\
\hline MB 1352 & meningitis & relevant & 6 & - & - & - & 1104 & $n d f^{c}$ & ungroupable $^{d}$ & + & - & - & - & non-disease-associated \\
\hline PP 386 & meningitis & relevant & 6 & - & - & - & 1104 & $n d f^{c}$ & ungroupable $^{d}$ & + & - & - & - & non-disease-associated \\
\hline MB 1050 & meningitis & relevant & 6 & - & - & - & 1104 & $\operatorname{ndf}^{c}$ & ungroupable $^{d}$ & + & - & - & - & non-disease-associated \\
\hline PP 504 & septicemia & relevant & 6 & - & - & - & 1104 & ndfc & ungroupable ${ }^{d}$ & + & - & - & - & non-disease-associated \\
\hline MB 185 & polyarthritis & relevant & 6 & - & - & - & 1104 & ndf $^{c}$ & ungroupable $^{d}$ & + & - & - & - & non-disease-associated \\
\hline SS 1364 & septicemia & relevant & 6 & - & - & - & 1104 & ndf $^{c}$ & ungroupable $^{d}$ & + & - & - & - & non-disease-associated \\
\hline SS 3896 & septicemia & relevant & 6 & - & - & - & 1104 & $n d f c$ & ungroupable $^{d}$ & + & - & - & - & non-disease-associated \\
\hline PP 438 & meningitis, polyarthritis & relevant & 6 & - & - & - & 1104 & $n f^{c}$ & ungroupable $^{d}$ & + & - & - & - & non-disease-associated \\
\hline PP 693 & polyarthritis, septicemia & relevant & 6 & - & - & - & 1104 & ndfc & ungroupable $^{d}$ & + & - & - & - & non-disease-associated \\
\hline MB 1593 & meningitis & relevant & 7 & - & - & sly & 1130 & CC87 & 2 & + & - & + & + & non-disease-associated \\
\hline MB 582 & meningitis & relevant & 7 & - & $m r p^{* * *}$ & - & 29 & $\mathrm{CC} 28$ & 4 & + & - & $+*$ & - & disease-associated \\
\hline MB 841 & meningitis, polyarthritis & relevant & 7 & - & $m r p^{* * *}$ & - & 29 & $\mathrm{CC} 28$ & 4 & + & - & $+^{*}$ & - & disease-associated \\
\hline MB 36 & septicemia & relevant & 7 & - & $m r p^{* * *}$ & - & 29 & $\mathrm{CC} 28$ & 4 & + & - & $+^{*}$ & - & disease-associated \\
\hline PP 478 & emaciation, colibacillosis & not relevant & 8 & - & - & sly & 1109 & CC1109 & $\mathrm{N}$ & + & - & $+*$ & - & disease-associated \\
\hline PP 283 & polyarthritis & not relevant & 8 & - & - & sly & 1106 & CC1109 & N & + & - & $+^{*}$ & + & non-disease-associated \\
\hline MB 386 & septicemia & not relevant & 8 & - & - & sly & 87 & CC87 & 2 & + & - & + & + & non-disease-associated \\
\hline PP 789 & septicemia & relevant & 9 & - & - & sly & 1109 & CC1109 & $\mathrm{N}$ & + & - & $+^{*}$ & - & disease-associated \\
\hline MB 1452 & meningitis & relevant & 9 & - & - & sly & 231 & CC1109 & $\mathrm{N}$ & + & - & $+*$ & - & disease-associated \\
\hline MB 1497 & septicemia & not relevant & 9 & - & - & sly & 1237 & CC1237 & novel1e & + & - & - & - & non-disease-associated \\
\hline
\end{tabular}


Table 2 (continued)

\begin{tabular}{|c|c|c|c|c|c|c|c|c|c|c|c|c|c|c|}
\hline PP 421 & meningitis & relevant & 9 & - & - & - & 1105 & $\mathrm{~S}^{\mathrm{c}}$ & $7-3$ & + & - & $+*$ & - & disease-associated \\
\hline MB 706 & meningitis & relevant & 9 & - & - & - & 1105 & $\mathrm{Sc}^{\mathrm{c}}$ & $7-3$ & + & - & $+^{*}$ & - & disease-associated \\
\hline PP 203 & meningitis & relevant & 9 & - & - & - & 1105 & $\mathrm{~S}^{\mathrm{c}}$ & $7-3$ & + & - & $+^{*}$ & - & disease-associated \\
\hline PP 536 & septicemia & relevant & 9 & - & - & - & 1105 & $\mathrm{Sc}^{c}$ & $7-3$ & + & - & $+^{*}$ & - & disease-associated \\
\hline PP 722 & septicemia & relevant & 9 & - & - & - & 1105 & $\mathrm{~S}^{\mathrm{c}}$ & $7-3$ & + & - & $+^{*}$ & - & disease-associated \\
\hline PP 742 & meningitis & relevant & 9 & - & - & - & 1105 & $\mathrm{Sc}^{\mathrm{c}}$ & 7-3 & + & - & $+*$ & - & disease-associated \\
\hline MB 923 & meningitis & relevant & 9 & - & - & - & 1105 & $\mathrm{sc}^{\mathrm{c}}$ & $7-3$ & + & - & $+^{*}$ & - & disease-associated \\
\hline SS 3878 & polyarthritis, peritonitis & not relevant & 9 & - & - & - & 1236 & CC1237 & novel1 ${ }^{\mathrm{e}}$ & + & - & - & - & non-disease-associated \\
\hline PP 186 & acute circulation shock & not relevant & 12 & - & - & - & 1099 & $\mathrm{Sc}^{\mathrm{c}}$ & ungroupable $^{d}$ & + & - & - & - & non-disease-associated \\
\hline MB 1493 & pneumonia & not relevant & 12 & - & - & - & $n d^{b}$ & - & ungroupable $^{d}$ & + & - & - & - & non-disease-associated \\
\hline PP 728 & $\begin{array}{l}\text { osteochondrosis } \\
\text { dissecans }\end{array}$ & not relevant & 15 & epf & $m r p^{* * *}$ & sly & 1110 & $\mathrm{~S}^{\mathrm{c}}$ & $N$ & + & - & - & - & non-disease-associated \\
\hline PP 402 & polyarthritis, septicemia & not relevant & 15 & - & $m r p^{*}$ & sly & 1134 & $\mathrm{sc}^{\mathrm{c}}$ & 1 & + & - & - & + & non-disease-associated \\
\hline MB 979 & placentitis, pneumonia & relevant & 15 & - & - & sly & 1115 & $\mathrm{~S}^{\mathrm{c}}$ & 1 & + & - & - & + & non-disease-associated \\
\hline PP 429 & dermatitis & not relevant & 16 & - & - & - & 1107 & $n d f c$ & $\mathrm{~N}$ & + & - & - & - & non-disease-associated \\
\hline MB 1047 & septicemia & relevant & 16 & - & - & - & 1239 & $\mathrm{Sc}^{\mathrm{c}}$ & 7-3 & + & - & - & - & non-disease-associated \\
\hline SS 4339 & pneumonia, colitis & not relevant & 16 & - & - & - & 1127 & $\mathrm{~S}^{\mathrm{c}}$ & $\mathrm{N}$ & + & - & - & - & non-disease-associated \\
\hline PP 263 & septicemia & relevant & 16 & - & - & - & 1121 & $\mathrm{Sc}^{\mathrm{c}}$ & $7-3$ & + & - & - & - & non-disease-associated \\
\hline MB 126 & no gross lesions & not relevant & 16 & - & - & - & 1111 & $\mathrm{ndf}^{\mathrm{c}}$ & $N$ & + & - & - & - & non-disease-associated \\
\hline MB 1091 & pneumonia, septicemia & relevant & 16 & - & - & - & 1116 & $\mathrm{ndfc}^{\mathrm{c}}$ & $\mathrm{N}$ & + & - & - & - & non-disease-associated \\
\hline PP 106 & meningitis, pneumonia & relevant & 19 & - & - & - & 1128 & $\mathrm{sc}^{\mathrm{c}}$ & novel1e & + & - & - & - & non-disease-associated \\
\hline PP 172 & $\begin{array}{l}\text { endocarditis valvularis, } \\
\text { pneumonia }\end{array}$ & relevant & 28 & - & - & sly & 1100 & $\mathrm{sc}^{\mathrm{c}}$ & $\mathrm{N}$ & + & - & - & - & non-disease-associated \\
\hline SS 2739 & pneumonia & not relevant & 28 & - & $m r p^{*}$ & sly & 1118 & $\mathrm{~S}^{c}$ & $\mathrm{~N}$ & + & - & - & - & non-disease-associated \\
\hline PP 560 & meningitis & relevant & 31 & - & - & - & 1122 & $\mathrm{sc}^{\mathrm{c}}$ & $\mathrm{N}$ & + & - & - & + & non-disease-associated \\
\hline MB 1297 & endocarditis valvularis & relevant & 31 & - & - & - & 1125 & $\mathrm{~S}^{\mathrm{c}}$ & novel4e & + & - & - & - & non-disease-associated \\
\hline SS 3726 & $\begin{array}{l}\text { meningitis, pneumonia, } \\
\text { septicemia }\end{array}$ & relevant & $N T^{a}$ & - & - & - & 1244 & $\mathrm{~S}^{\mathrm{c}}$ & ungroupable $^{d}$ & + & - & - & - & non-disease-associated \\
\hline PP 216 & septicemia & relevant & $N T^{a}$ & - & - & - & 1120 & $\mathrm{Sc}^{\mathrm{c}}$ & ungroupable $^{d}$ & + & - & - & - & non-disease-associated \\
\hline PP 464 & pneumonia & not relevant & $N T^{a}$ & - & - & - & 1108 & $\mathrm{~S}^{\mathrm{c}}$ & novel1 ${ }^{\mathrm{e}}$ & + & - & - & - & non-disease-associated \\
\hline PP 715 & pneumonia, polyarthritis & not relevant & $N T^{a}$ & - & - & - & 1123 & $\mathrm{Sc}^{\mathrm{c}}$ & ungroupable $^{d}$ & + & - & - & - & non-disease-associated \\
\hline PP 262 & pneumonia, septicemia & not relevant & $N T^{a}$ & - & - & - & $n d^{b}$ & - & ungroupable $^{d}$ & + & - & - & - & non-disease-associated \\
\hline MB 810 & pneumonia & not relevant & $N T^{a}$ & - & - & - & 1243 & $\mathrm{~S}^{\mathrm{c}}$ & ungroupable $^{d}$ & + & - & - & - & non-disease-associated \\
\hline
\end{tabular}

Isolates were chosen based on the fact of an unequivocal link of etiologically relevant S. suis diseased or non-disease-associated pigs. Pathotyping results, which did not correspond to the results obtained by histopathological findings, are indicated in red.* indicates two isolates, which were included in the evaluation although a record of histopathological findings was missing, but due to evident bacteriological findings and appropriate anamnesis, strains were evaluated as relevant. mrp variants identified: $m r p^{5}$ (747 bp), $m p r(1148 \mathrm{bp}), m r p^{*}(1556 \mathrm{bp}), m r p^{* * *}(2400 \mathrm{bp})$. epf* represents a large variant of epf, which was detected only in one isolate (191715-1). + ${ }^{*}$ relates to the pathotyping tool representing a variant PCR product of copper exporting ATPase gene.

a Nontypeable.

b Not determined (nd): one lacking housekeeping gene.

c No clonal complex could be assigned for ST that occurred as singletons (S) or with no determined founder (ndf).

d Ungroupable: allele missing.

e Novel: allel combination not described for MCG sequencing typing by Zheng et al. [42].

f Wileman et al. [31].

within two different years. However, the majority of isolates, representing $43.2 \%$ of the tested collective, was isolated uniquely in 1 year.

\section{Association between genotype and CCs}

STs of all S. suis isolates were assigned to CCs using the eBurst illustration (Figure 3). Among the 88 isolates analyzed, eight $\mathrm{CCs}$ were identified: $\mathrm{CC} 1, \mathrm{CC} 17, \mathrm{CC} 28$, CC87, CC13/149, CC1109, CC1112, and CC1237 comprising $3,3,8,2,6,7,1$, and 5 isolates, respectively. The 
distribution of $S$. suis isolates in relation to ST revealed ST1104 (cps6, $n=13 / 13$ ) with the highest prevalence of $14.8 \%$ and no association to any CCs. Further on, $9.1 \%$ of isolates representing ST1105 (cps9, $n=8 / 14$ ) were found to be unrelated singleton-isolates not associated to any CCs.

A total of $6.8 \%$ of isolates belonged to CC13/149 harboring ST13 (cps1, $n=6 / 6)$. 3.4\% of isolates belonged to CC28 comprising ST29 (cps7, $n=3 / 4$ ). ST17, ST28, ST231, ST1103, and ST1236 represented each $3.4 \%$ of isolates associated with CC17, CC28, CC1109, CC1, and $\mathrm{CC} 1237$, respectively. Finally, all remaining isolates had different STs only occurring in few isolates illustrating a massive genetic diversity identified in diseased Swiss pigs (Additional file 3). Samples comprising CC1 included ST1103 (cps2, $n=3 / 4$ ). ST17 (cps4, $n=3 / 3$ ) was part of CC17. The most abundantly identified CC28 contained isolates of ST28 (cps $1 / 2, n=2 / 3$; cps $2, n=1 / 4)$, ST29 (cps7, $n=3 / 4)$, ST856 (cps3, $n=1 / 4)$, and ST1133 (cps1/2, $n=1 / 3$ ). CC87 contained ST87 (cps8, $n=1 / 3$ ) and ST1130 (cps7, $n=1 / 4$ ). All isolates of ST13 (cps1, $n=6 / 6)$ were part of CC13/149. CC1109 comprised isolates of ST231 (cps3, $n=2 / 4 ; c p s 9, n=1 / 14), \mathrm{ST} 1106$ (cps8, $n=1 / 3)$, ST1109 (cps8, $n=1 / 3 ;$ cps $9, n=1 / 14)$, and ST1113 (cps3, $n=1 / 4$ ). CC1112 harbored only one isolate of ST1112 (cps15, $n=1 / 6)$. Finally, CC1237 comprised ST1236 (cps9, $n=3 / 14)$, ST1237 (cps9, $n=1 / 14$ ) and ST1240 (cps21, $n=1 / 2)$ (Table 3).

\section{Virulence profiling}

$\mathrm{CC} 1$ harbored one cps 2 isolate with a large variant of epf: $\left(m r p^{+} e p f s l y^{+}\right)$, whereas two cps 2 isolates had a $m r p^{+} e p f^{-} s y^{+}$genotype. Variable subtypes of $m r p$ were identified in one cps 2 isolate and two $c p s 1 / 2$ isolates with a genotype of $m r p^{\mathrm{S}} e p f^{-} s l y^{-}$; one $c p s 1 / 2$ isolate, one cps 3 isolate, and three cps 7 isolates contained $m r p^{* * *} e p f^{-} s l y^{-}$. Furthermore, genotype $m r p^{\mathrm{S}} e p f^{+} s l y^{+}$ was found in all three isolates of cps 4 belonging to CC17. Two isolates of $c p s 15$ had a $m r p^{* * *} e p f^{+} s l y^{+}$ genotype, whereas one isolate of each cps 15 and cps 28 contained mrp"epf sly $^{+}$. 22.7\% of isolates showed a $m r p^{-} e p f^{-} s y^{+}$genotype, which was associated with CC13/149 consisting of cps $1(n=6 / 6)$; CC1109 comprising cps $3(n=3 / 4)$, cps $8 \quad(n=2 / 3)$, and $c p s 9$ $(n=2 / 14)$; CC87 consisting of cps $7(n=1 / 4)$, and cps 8 ( $n=1 / 3)$; CC1237 comprising cps $9(n=1 / 14)$; and ultimately, singleton-isolates consisting of $\operatorname{cps} 15(n=3 / 6)$ and cps $28(n=1 / 2)$. Finally, a total of $56.8 \%$ of isolates studied had a $m r p^{-} e p f^{-} s l y^{-}$genotype, which could not be associated to any CCs and were identified as singletons consisting of cps5 $(n=2 / 2), \operatorname{cps} 6(n=13 / 13)$, cps $9(n=11 / 14)$, cps $12(n=3 / 3), \operatorname{cps} 16(n=6 / 6), \operatorname{cps} 19$ $(n=1 / 1), \quad \operatorname{cps} 21 \quad(n=2 / 2), \operatorname{cps} 31 \quad(n=3 / 3)$, and NT $(n=9 / 9)$ (Additional file 2).

\section{MCG typing}

MCG typing revealed MCG group 1 for members of CC1, CC17, and CC1112; MCG group 2 for isolates of CC87; MCG group 4 for isolates of CC28; MCG group $\mathrm{N}$ (representing an ungroupable subset of isolates) for CC13/149 and CC1109; and a novel MCG group for isolates of CC1237. In total, $13.6 \%$ of isolates were classified into five novel MCG groups not previously described (Table 4). Almost $30 \%$ of isolates could not be assigned to any MCG group due to the failure of one housekeeping gene identification.

\section{Pathotyping for differentiation between invasive disease-associated and non-disease-associated isolates}

Figure 4 shows the amplicon patterns obtained by multiplex PCR after capillary electrophoresis. Amplicons of S. suis specific genetic marker (SSU0577) were obtained by all isolates proving the correct species identification. Amplicons of 892 bp represented the putative sugar $A B C$ reporter as a non-disease-associated marker (SSUT30534), whereas disease-associated gene markers were identified by amplicons of $347 \mathrm{bp}$ and 211 bp illustrating a type I RM system S protein (SSU1589) and a predicted copper ATPase (SSU0207), respectively. Additionally, new variant forms of the copper ATPase gene marker appeared, illustrated by a 190 bp long amplicon (Additional file 4). In total, $29.3 \%(n=22 / 75)$ of isolates analyzed harbored a gene variant of the copper ATPase gene containing partial deletions, represented by all isolates of CC28 $(n=7 / 75)$, CC1109 $(n=7 / 75)$, and cps 9 isolates with ST1105 $(n=8 / 75)$. As a contrast, $4 \%(n=3 / 75)$ of isolates belonging to $\mathrm{CC} 1$ showed a $211 \mathrm{bp}$ long amplicon as comprehended by the virulent P1/7 strain (Table 2). The disease-associated marker type I RM system S protein-gene revealed a PCR amplification of $347 \mathrm{bp}$ only for the three isolates comprising $\mathrm{CC} 1$. Sequence analysis of some exemplary isolates with $c p s 1 / 2$, cps 1 , and cps 2 revealed a truncated gene version of the implicated gene, whereas the reverse primer could not bind to the target sequence due to the absence of the $3^{\prime}$ end of the gene in most isolates analyzed (Additional file 4 ).

Pathotyping of the subset of 75 isolates unequivocally attributed as $S$. suis relevant or not $S$. suis relevant, revealed isolates of cps 2 and cps 9 to be in accordance with the pathotyping tool. However, comparing S. suis relevant isolates of cps 1 and cps 6 to results obtained by pathotyping, all isolates yielded a contradictory result. All other cps types could not clearly be classified by 


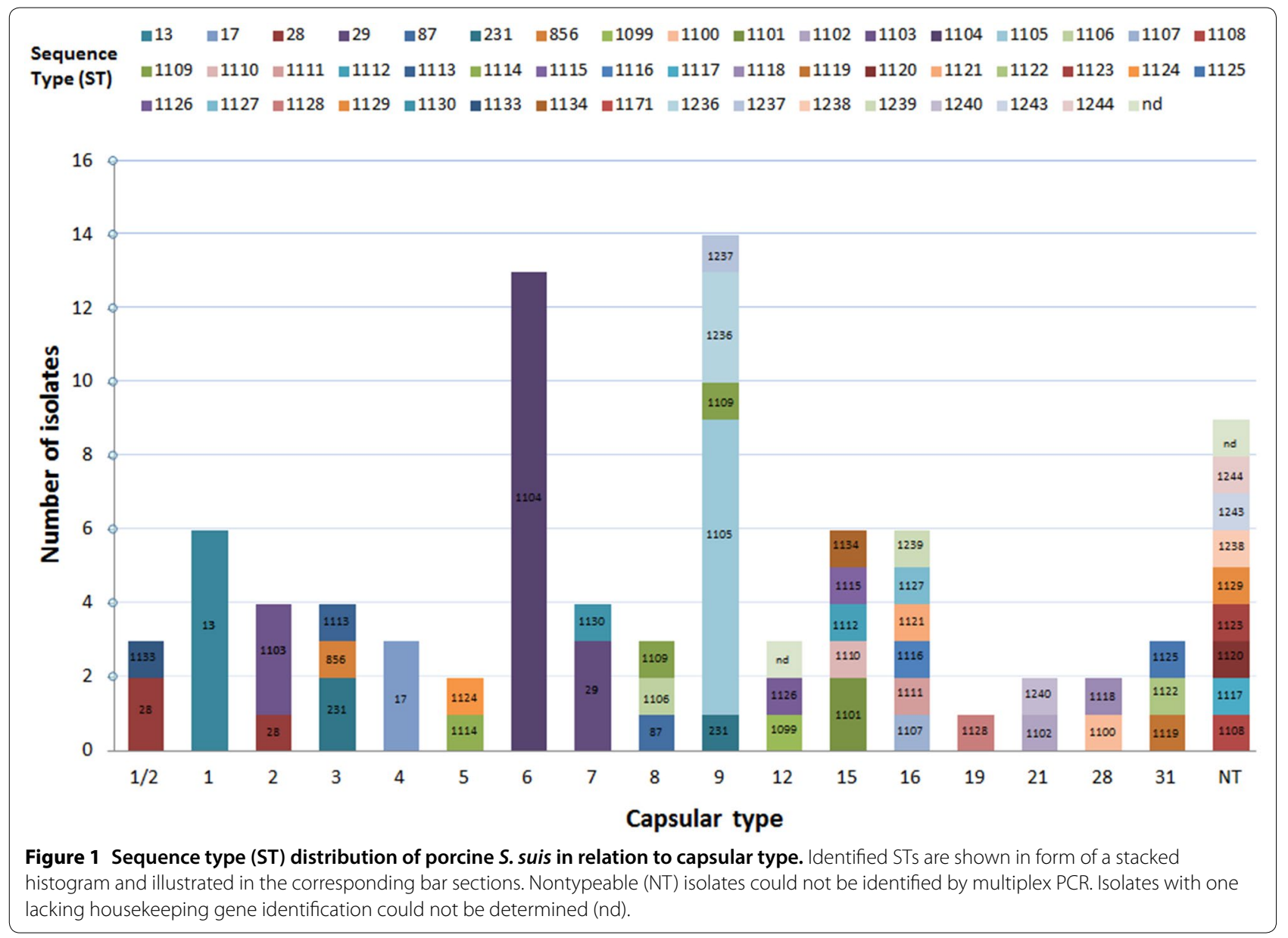

pathotyping due to the paucity of isolates having the same capsular type and therefore no statement can be made.

\section{Discussion}

In this study a detailed assessment of genetic characteristics of Swiss S. suis isolates of diseased pigs was performed and compared to an earlier analyzed human isolate. In general, seventeen different cps types were identified underlining high serotype diversity in accordance to a previously observed massive diversity in whole genome sequences of a chosen set of isolates of clinically affected pigs [43]. The most common cps types found among the examined strain collection were cps 9 and cps6. Contrary to a German investigation with over $700 \mathrm{~S}$. suis isolates, not a single cps6 strain was found, which is worth to highlight when keeping in mind that Germany is a main neighboring country of Switzerland [44].

In the current report, all 13 isolates identified as cps6 comprised a single clone of ST1104, which was not associated to any CCs. ST1104 is representing a clone with no determined founder, identified to be a single locus variant (SLV; differing only at cpn60) of a Danish invasive S. suis strain with ST55 [45]. Noteworthy, this identified clone reoccurred throughout eight different years (Figure 2) underlining its capacity of causing disease in pigs due to an invasive behavior of the involved strain. Interestingly, a study from Chile, investigating phenotypically and genotypically diseased pigs at nonrelated farms during the time span of 2007-2011, revealed a single clone of S. suis with $n=28 / 29$ of isolates identified to be serotype 6. Although an invasive phenotype could be observed, no enhanced virulence could be proven in a murine model testing serotype 6 isolates from Chile including the Danish serotype 6 reference strain. No concomitant infections of the involved isolates could be observed, therefore, an enhanced virulence capacity was suggested [46].

Furthermore, it was notable that all isolates belonging to cps9 were rather heterogenic harboring five different STs, although one abundant ST (ST1105, $n=8 / 14$ ) seemed to be predominant among the identified samples. The genetic diversity of $c p s 9$ isolates in China and Canada showed a high variety among examined isolates $[47$, 


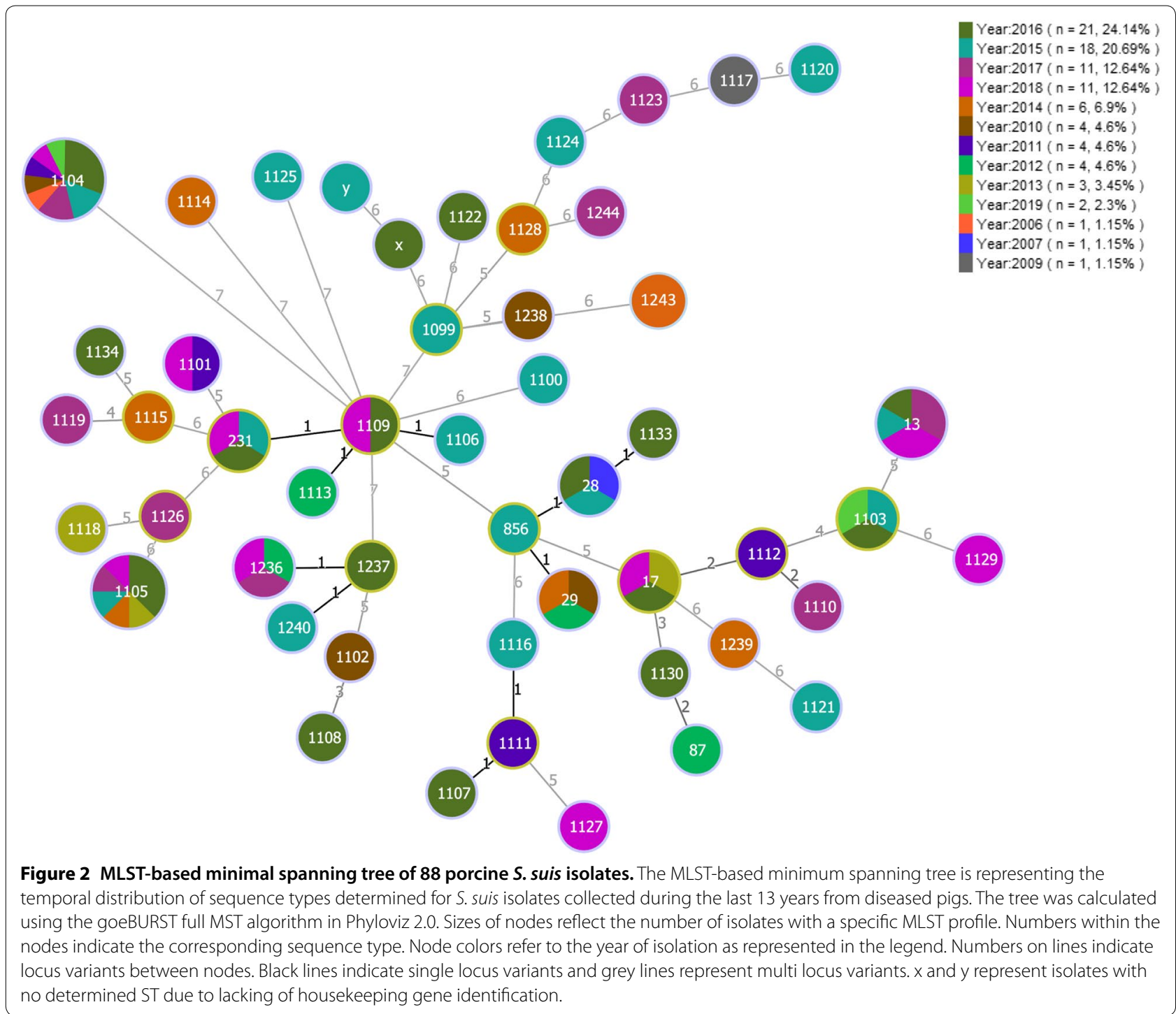

48], which is comparable to the observed genetic diversity of inspected Swiss isolates. Interestingly, the aforementioned clone comprising ST1105 reoccurred within six different years (Figure 2) emphasizing its importance among the pig population in Switzerland and its ability to induce infections. Similar reports of pigs with invasive disease have stated a predominance of $m r p^{*} c p s 9$ belonging to CC16/CC87 for Europe including The Netherlands, Spain, Germany and Belgium $[9,16]$. The observation that cps 9 isolates of the present study did not harbor $m r p$ together with the identification of novel sequence types, underlines the emergence of very unique $S$. suis strains with new genetic rearrangements rarely found in other countries.

Actually, Switzerland has very little traffic of piglets with foreign countries and the pig farming system is different in comparison to other European countries. Herds are much smaller and the utilization of farrowing crates for reproduction purposes is prohibited by law since 1997. The physiological behavior in nesting is impaired due to the narrowing conditions in farrowing crates and consequently, a more natural environment helps to avoid complications during birth of piglets thereby strengthening their immune status. In addition, Switzerland is considered to be free of porcine reproductive and respiratory syndrome virus (PRRSV) [49] and Mycoplasma hyopneumoniae [50]. Moreover, a majority of farms vaccinate piglets against porcine circovirus (PCV) [51]. The general health status of Swiss pigs is considered to be high, which could be explained by the absence of these pathogens considered to be immunosuppressive and therefore having an implication in the defense of S. suis. Nevertheless, stress caused by weaning at an age of 28 days and rehousing of piglets usually after $10-12$ weeks frequently paired 


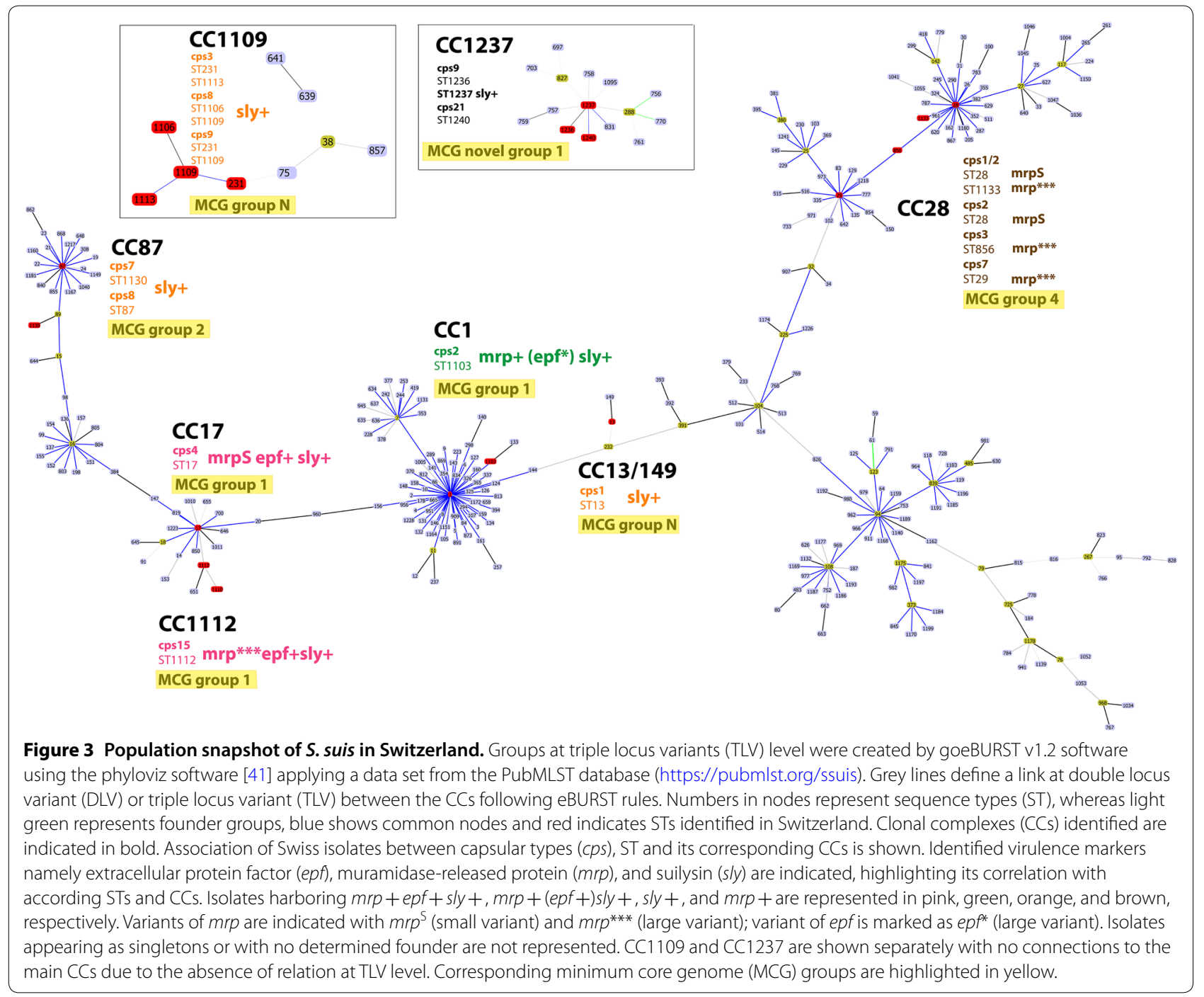

with a change of feed at the fattening farms can lead to infection caused by virulent $S$. suis strains. Fattening farms usually combine weaning piglets of different farms leading to a higher transmission rate of S. suis among piglets. These circumstances could explain the high genetic variability and the distinct composition regarding ST and capsular types of $S$. suis strains obtained in this study in contrast to other countries.

Most remarkably, isolates belonging to $\mathrm{CC} 1$ are considered as potentially zoonotic and were mostly described as virulent strains [26]. In the present study three out of four isolates comprising cps 2 were identified belonging to $\mathrm{CC} 1$. Showing its current relevance, one cps 2 isolate originating from a brain of a diseased pig, was collected very recently in fall 2019, highlighting an emerging S. suis clone with ST1103. Interestingly, the implicated strain had a $m r p^{+} e p f^{*} s l y^{+} c p s 2$ genotype containing a large variant of epf, which is considered to be a moderately virulent pathotype [21]. In fact, this specific clone is a SLV of ST1, differing only in allele $g k i$. The fourth cps 2 isolate had a more common ST28 belonging to CC28. One highly virulent human ST1 (cps14) isolate from another study [52] was also part of $\mathrm{CC} 1$ harboring $m r p^{+} e p f^{+} s l y^{+}$underlining an association between virulence-associated markers epf, mrp, and sly with STs rather than cps types (Figure 3). Three cps 2 isolates (ST1103) were classified as MCG group 1, whereas the fourth cps2 isolate (ST28) was part of MCG4. For the human isolate, MCG typing revealed a novel group not described before, which was uniquely attributed to this isolate. The fact that this isolate was classified as a novel MCG group, absent among the analyzed Swiss porcine isolates, proves an improbable transmission event of this highly virulent pathogenic isolate from Swiss pigs pointing out a different source of infection. The fact that the virulent 


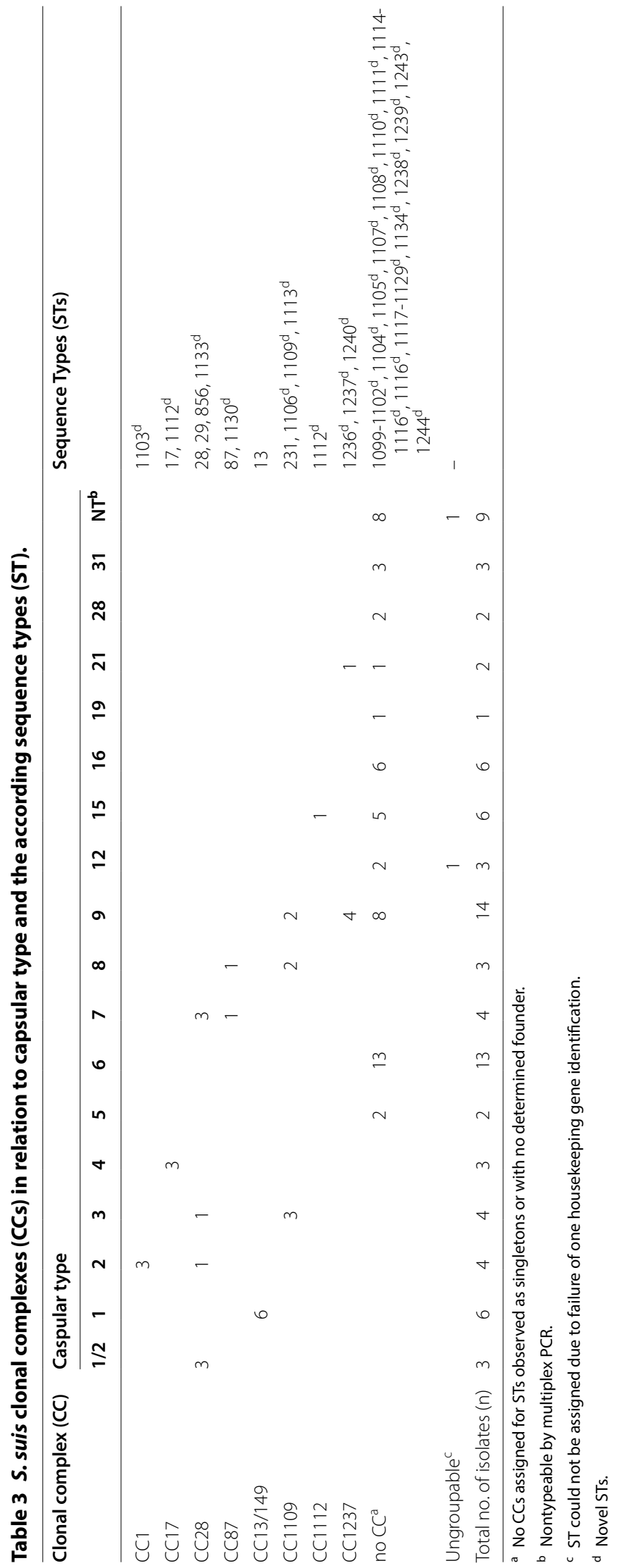


Table 4 Single nucleotide polymorphisms (SNPs) used for minimum core sequencing (MCG).

\begin{tabular}{|c|c|c|c|c|c|c|c|c|c|c|c|c|c|c|c|c|}
\hline $\begin{array}{l}\text { Position } \\
\text { in GZ1 } \\
\text { genome }^{\mathrm{a}}\end{array}$ & $\mathrm{E}^{\mathrm{b}}$ & $\begin{array}{c}\text { Group } \\
1\end{array}$ & $\begin{array}{c}\text { Group } \\
2\end{array}$ & $\begin{array}{c}\text { Group } \\
\mathbf{3}\end{array}$ & $\begin{array}{c}\text { Group } \\
4\end{array}$ & $\begin{array}{c}\text { Group } \\
5\end{array}$ & $\begin{array}{c}\text { Group } \\
6\end{array}$ & $\begin{array}{c}\text { Group } \\
7-1\end{array}$ & $\begin{array}{c}\text { Group } \\
7-2\end{array}$ & $\begin{array}{c}\text { Group } \\
7-3\end{array}$ & $\mathbf{N}^{c}$ & novel1 ${ }^{d}$ & novel $2^{\mathrm{e}}$ & novel3 ${ }^{f}$ & novel4g & novel5 ${ }^{\mathrm{h}}$ \\
\hline 2028696 & A & G & G & G & G & G & G & G & G & G & G & G & G & G & G & G \\
\hline 2028744 & A & A & G & G & G & G & G & G & G & G & G & G & $A$ & A & G & G \\
\hline 824818 & $\mathrm{~T}$ & $\mathrm{~T}$ & C & $\mathrm{T}$ & $T$ & $\mathrm{~T}$ & $\mathrm{~T}$ & $\mathrm{~T}$ & $\mathrm{~T}$ & $\mathrm{~T}$ & $\mathrm{~T}$ & $\mathrm{~T}$ & $\mathrm{~T}$ & $\mathrm{~T}$ & C & $\mathrm{T}$ \\
\hline 822644 & $C$ & C & C & $\mathrm{T}$ & C & C & $C$ & C & C & C & $\mathrm{C}$ & C & $C$ & C & C & C \\
\hline 825000 & G & G & G & G & G & A & G & G & G & G & G & G & G & G & G & G \\
\hline 81999 & G & G & G & G & G & G & $\mathrm{T}$ & G & G & G & G & $\mathrm{T}$ & G & G & G & G \\
\hline 81404 & $A$ & A & A & A & A & A & A & G & A & A & $A$ & A & G & A & A & A \\
\hline 81419 & $\mathrm{~T}$ & $\mathrm{~T}$ & $\mathrm{~T}$ & $\mathrm{~T}$ & $\mathrm{~T}$ & $\mathrm{~T}$ & $\mathrm{~T}$ & $\mathrm{~T}$ & G & $T$ & $\mathrm{~T}$ & $\mathrm{~T}$ & $\mathrm{~T}$ & $\mathrm{~T}$ & $\mathrm{~T}$ & G \\
\hline 572576 & $\mathrm{G}$ & G & $\mathrm{G}$ & G & G & G & G & G & G & A & $\mathrm{G}$ & A & G & G & A & A \\
\hline
\end{tabular}

MCG groups as defined by Zheng et al. [42] including 5 novel MCG groups identified in the present study. Red highlights discriminative SNPs for each SNP profile.

a Reference genome: strain GZ1 (GenBank accession number: CP000837).

b Epidemic (E) strains (ST7 strains) identified by Zheng et al. [42].

c Ungroupable.

The following isolates resulted in novel MCG groups with a new SNP profile.

d MB1158 (cps type 5), MB1497, SS2097 (cps type 9), PP106 (cps type 19), MB1185 (cps type 21), SS921 (nontypeable).

e MB1629 (cps type 12).

f Human pathogen strain (cps type 14).

g MB 1297 (cps type 31).

h SS 3919 (cps type 31).

strain was introduced through a person originating from Moldova importing meat to Switzerland [52], proves an independent introduction of this zoonotic strain. Furthermore, all cps4 isolates which are part of CC17 were classified as MCG group 1 with a genotype of $m r p^{\mathrm{S}} e p f^{+} s l y^{+}$emphasizing a possible increased virulence potential. In addition, a majority of $\operatorname{cps} 15$ isolates belonged to MCG group 1, whereas two isolates had a $m r p^{* * *} e p f^{+} s l y^{+}$genotype. Moreover, most isolates of cps7 were ST29 and belonged to CC28, similar to an emerging virulent strain recently reported in Germany [53] highlighting that isolates classified to be part of CC28 clearly show invasive and highly virulent features.

To evaluate the usefulness of pathotyping [31], a subset of 75 isolates having an unequivocal link of etiologically relevant $S$. suis diseased or non-disease-associated pigs was screened by the pathotyping tool. Cps 2 and cps 9 showed a clear correlation between pathotyping results and histopathological findings. Remarkably, comparing observed phenotypes classified to be $S$. suis relevant, obtained by a combination of histopathological examinations and bacteriological analysis, to results obtained by pathotyping, isolates of cps 1 and cps 6 yielded a contradictory result since no gene marker indicating disease-association yielded a positive PCR result. Due to the perceived inconsistency of phenotypically observed manifestations determined to be $S$. suis relevant in contrast to the non-S. suis relevant results obtained with the pathotyping tool, sequence analysis of the involved gene markers (type I RM systems S protein- and copper ATPase-gene) was performed highlighting a high genetic variability of different isolates (Additional file 4). Worth mentioning, all cps type 1 isolates (ST13) were associated to CC13/149. Interestingly, in two previous studies [29, 54 isolates of ST13 were associated with a pathogenic phenotype and represented an indicator of virulence underlining its importance. Yet undiscovered potential virulence factors especially of cps 1 and cps 6 strains remain matter of further investigation.

Cps3, cps7, cps 15 , and cps 16 showed partially contradictory results between histopathological findings and the pathotyping tool, which points out the difficulty of consistent histopathological examinations, considering the multifaceted properties of bacterial infections.

\section{Conclusion}

With this study an expanded knowledge about the population structure and the genetic diversity of $S$. suis could be achieved. The epidemiological situation of S. suis in Switzerland revealed a heterogeneous composition of strains with high genetic variability belonging to several CCs. Strikingly, cps 9 and cps 6 were among the predominant capsular types associated with $S$. suis diseased pigs. Genetic variant forms of implicated disease-associated genes can be explained partially with frequent genomic rearrangements in S. suis as shown previously [27, 55]. 


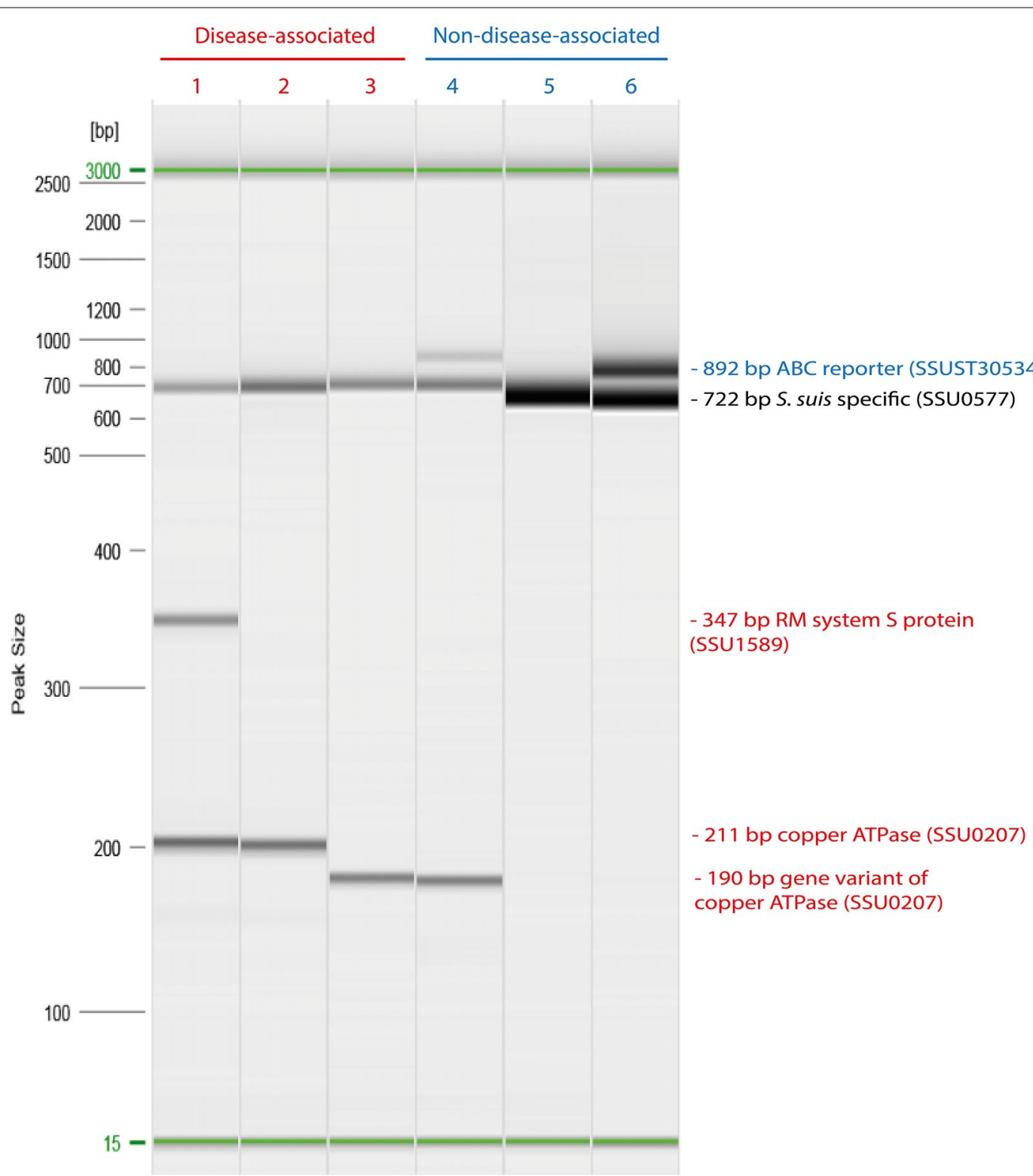

Figure 4 Capillary electrophoresis plots illustrating invasive disease-associated and non-disease-associated Swiss S. suis isolates.

On the left hand side the DNA size marker (100 bp-2.5 kb) is shown. The alignment marker (green) is representing the start and end of electrophoresis. Amplicons sizes obtained from the molecular pathotyping tool [31] are indicated on the right hand side. Lane 1 corresponds to the amplification reaction of a highly virulent isolate of cps type 2 (sequence type 1103) revealing both disease-associated markers (red) predicting a putative copper exporting ATPase 1 (SSU0207) and a type I restriction-modification (RM) system S protein (SSU1589). Lane 2 and 3 represent disease-associated isolates harboring the copper ATPase and a variant form with a 21 base pair (bp) deletion (lane 3), respectively. Lane 4, 5, and 6 demonstrate observed amplification patterns of non-disease-associated samples (blue). Lane 4 shows a PCR amplicon for the putative sugar ABC reporter (SSUST30534) also in the presence of the copper ATPase gene. Samples with no amplification of any markers are considered to be non-disease-associated (lane 5). A sporulation regulator (WhiA) serves as identification control for S. suis (SSU0577).

These genetically variable and distinct rearrangements could be facilitated by the unique pig farming of Switzerland with very little transfer of piglets from other European countries, however, with a frequent transport rate of weaning piglets to fattening farms.
In view of an affordable tool as a diagnostic application to rapidly discover highly invasive $S$. suis strains and to highlight a potentially threatening zoonotic pathogen, it would be advantageous to have a reliable pathotyping tool. The screening using our well characterized strain collection showed a good feasibility of the tool for cps 2 
and $\operatorname{cps} 9$, however, for cps 1 and $\operatorname{cps} 6$ contradictory results were obtained. Therefore, we recommend using the pathotyping tool only in case of cps2- and cps9-identification in order to differentiate between invasive diseaseassociated isolates and non-disease-associated isolates.

An efficient identification of highly virulent strains could help to alert public health surveillance programs to take action in line with a one health approach. Keeping track of disease-associated isolates helps to reduce the risk of zoonotic infections and will allow promoting a high standard in animal welfare programs.

\section{Supplementary information}

Supplementary information accompanies this paper at https://doi. org/10.1186/s13567-020-00813-w.

Additional file 1. Capsular type distribution among Swiss S. suis isolates. Number of isolates in relation to capsular type is indicated. $\mathrm{NT}=$ nontypeable due to no successful capsular typing by multiplex PCR.

Additional file 2. Clinical data, genotyping, and determination of virulence markers of Swiss porcine $S$. suis isolates $(n=88)$.

Additional file 3. Distribution of porcine $S$. suis isolates in relation to sequence type and clonal complex.

Additional file 4. Alignment of target gene sequences and the corresponding amino acid sequences used by pathotyping. Sequence alignment of copper ATPase 1-gene (A and B) and partial gene sequence alignment of type I RM system $S$ protein-gene ( $C$ and $D$ ) of invasive disease-associated isolates of Swiss S. suis in comparison to the highly virulent reference strain $\mathrm{P} 1 / 7$ are shown visualizing different gene variants and its corresponding protein sequences. Conserved, matching nucleotide residues are illustrated as blue dots, whereas red represents differences of nucleotide sequences. (A) Copper ATPase 1-gene sequences of $S$. suis PP463 (cps2, ST28), SS470 (cps1/2, ST28), PP423 (cps1/2, ST1133), and PP536 (cPs9, ST1105) are represented. Primer sequences of the pathotyping tool are indicated in green. A duplication of a 54 bp long DNA segment in isolate SS470 and deletion of a 21 bp fragment in all represented Swiss isolates could be observed, illustrating a high genetic variability. (B) Corresponding amino acid sequence alignment of Copper ATPase 1 is shown. (C) RM system S protein gene sequences of S. suis PP463 (cps2, ST28), PP423 (cps1/2, ST1133), and PP269 (cps1, ST13) are represented. The forward primer is indicated in green, whereas the reverse primer could not be shown since illustrated Swiss isolates are truncated. (D) Corresponding amino acid sequence alignment of RM systems $S$ protein is shown.

\section{Abbreviations}

S. suis: Streptococcus suis; CPS: Capsular polysaccharides; MLST: Multilocus sequence typing; MCG: Minimum core genome; ST: Sequence type; CC: Clonal complex; EPF: Extracellular protein factor; MRP: Muramidase-released protein; SLY: Suilysin; RM: Restriction-modification; ABC: ATP-binding cassette; recN: Recombination/repair protein gene; $n$ : Number.

\section{Acknowledgements}

We would like to thank Robert Graage and Anja Kipar for the excellent support regarding clinical aspects about diseases in swine. We also wish to thank the lab staff of the Department of Veterinary Bacteriology, Vetsuisse Faculty, University of Zurich, for technical assistance.

\section{Authors' contributions}

SS, SP, GR, JS, MG and RS designed and coordinated the study. SS, GR, NSS and FR performed the experiments. MJAS, SP and SS analyzed data. SS and SP wrote the manuscript. RS, MG, GR and JS reviewed and edited the manuscript. All authors read and approved the final manuscript.

\section{Funding}

The authors have not declared a funding from any public or commercial agency.

\section{Availability of data and materials}

All data relevant to the study are included in the article or enclosed as additional files.

\section{Ethics approval and consent to participate}

In accordance with local legislation, ethical approval was not required and no animal experiments were carried out for this study.

\section{Competing interests}

The authors declare that they have no competing interests.

\section{Author details}

${ }^{1}$ Department of Veterinary Bacteriology, Institute for Food Safety and Hygiene, Vetsuisse Faculty, University of Zurich, Zurich, Switzerland. ${ }^{2}$ Institute for Veterinary Pathology, Vetsuisse Faculty, University of Zurich, Zurich, Switzerland. ${ }^{3}$ Bacteriology Laboratory, Geneva University Hospitals and University of Geneva, Geneva, Switzerland. ${ }^{4}$ Swine and Poultry Infectious Diseases Research Center (CRIPA), Groupe de recherche sur les maladies infectieuses des animaux de production (GREMIP), Faculty of Veterinary Medicine, University of Montreal, Saint-Hyacinthe, QC, Canada.

Received: 1 April 2020 Accepted: 15 June 2020

Published online: 08 July 2020

\section{References}

1. Gottschalk M, Segura M, Xu J (2007) Streptococcus suis infections in humans: the Chinese experience and the situation in North America. Anim Health Res Rev 8:29-45. https://doi.org/10.1017/s14662523070012 47

2. Arends JP, Hartwig N, Rudolphy M, Zanen HC (1984) Carrier rate of Streptococcus suis capsular type 2 in palatine tonsils of slaughtered pigs. J Clin Microbiol 20:945-947

3. Reams RY, Glickman LT, Harrington DD, Thacker HL, Bowersock TL (1994) Streptococcus suis infection in swine: a retrospective study of 256 cases. Part II. Clinical signs, gross and microscopic lesions, and coexisting microorganisms. J Vet Diagn Invest 6:326-334. https://doi.org/10.1177/10406 3879400600308

4. Segura M, Calzas C, Grenier D, Gottschalk M (2016) Initial steps of the pathogenesis of the infection caused by Streptococcus suis: fighting against nonspecific defenses. FEBS Lett 590:3772-3799. https://doi. org/10.1002/1873-3468.12364

5. Gottschalk M, Xu J, Calzas C, Segura M (2010) Streptococcus suis: a new emerging or an old neglected zoonotic pathogen? Future Microbiol 5:371-391. https://doi.org/10.2217/fmb.10.2

6. Wertheim HF, Nghia HD, Taylor W, Schultsz C (2009) Streptococcus suis: an emerging human pathogen. Clin Infect Dis 48:617-625. https://doi. org/10.1086/596763

7. Okura M, Osaki M, Nomoto R, Arai S, Osawa R, Sekizaki T, Takamatsu D (2016) Current taxonomical situation of Streptococcus suis. Pathogens. https://doi.org/10.3390/pathogens5030045

8. Baums CG, Valentin-Weigand P (2009) Surface-associated and secreted factors of Streptococcus suis in epidemiology, pathogenesis and vaccine development. Anim Health Res Rev 10:65-83. https://doi.org/10.1017/ s146625230999003x

9. Goyette-Desjardins G, Auger JP, Xu J, Segura M, Gottschalk M (2014) Streptococcus suis, an important pig pathogen and emerging zoonotic agent-an update on the worldwide distribution based on serotyping and sequence typing. Emerg Microbes Infec 3:e45. https://doi.org/10.1038/ emi.2014.45

10. Kerdsin A, Oishi K, Sripakdee S, Boonkerd N, Polwichai P, Nakamura S, Uchida R, Sawanpanyalert P, Dejsirilert S (2009) Clonal dissemination of human isolates of Streptococcus suis serotype 14 in Thailand. J Med Microbiol 58:1508-1513. https://doi.org/10.1099/jmm.0.013656-0 
11. Schultsz C, Jansen E, Keijzers W, Rothkamp A, Duim B, Wagenaar JA, van der Ende A (2012) Differences in the population structure of invasive Streptococcus suis strains isolated from pigs and from humans in The Netherlands. PLoS One 7:e33854. https://doi.org/10.1371/journ al.pone.0033854

12. Kerdsin A, Hatrongjit R, Gottschalk M, Takeuchi D, Hamada S, Akeda Y, Oishi K (2017) Emergence of Streptococcus suis serotype 9 infection in humans. J Microbiol Immunol 50:545-546. https://doi.org/10.1016/j. jmii.2015.06.011

13. Vecht U, Wisselink HJ, van Dijk JE, Smith HE (1992) Virulence of Streptococcus suis type 2 strains in newborn germfree pigs depends on phenotype. Infect Immun 60:550-556

14. Smith HE, Vecht U, Wisselink HJ, Stockhofe-Zurwieden N, Biermann $Y$, Smits MA (1996) Mutants of Streptococcus suis types 1 and 2 impaired in expression of muramidase-released protein and extracellular protein induce disease in newborn germfree pigs. Infect Immun 64:4409-4412

15. Jacobs AA, van den Berg AJ, Baars JC, Nielsen B, Johannsen LW (1995) Production of suilysin, the thiol-activated haemolysin of Streptococcus suis, by field isolates from diseased pigs. Vet Rec 137:295-296. https://doi. org/10.1136/vr.137.12.295

16. Wisselink HJ, Smith HE, Stockhofe-Zurwieden N, Peperkamp K, Vecht U (2000) Distribution of capsular types and production of muramidasereleased protein (MRP) and extracellular factor (EF) of Streptococcus suis strains isolated from diseased pigs in seven European countries. Vet Microbiol 74:237-248. https://doi.org/10.1016/s0378-1135(00)00188-7

17. Li Q, Fu Y, Ma C, He Y, Yu Y, Du D, Yao H, Lu C, Zhang W (2017) The nonconserved region of MRP is involved in the virulence of Streptococcus suis serotype 2. Virulence 8:1274-1289. https://doi.org/10.1080/21505 594.2017 .1313373

18. Pian Y, Wang P, Liu P, Zheng Y, Zhu L, Wang H, Xu B, Yuan Y, Jiang Y (2015) Proteomics identification of novel fibrinogen-binding proteins of Streptococcus suis contributing to antiphagocytosis. Front Cell Infect Mi 5:19. https://doi.org/10.3389/fcimb.2015.00019

19. Wang J, Kong D, Zhang S, Jiang H, Zheng Y, Zang Y, Hao H, Jiang Y (2015) Interaction of fibrinogen and muramidase-released protein promotes the development of Streptococcus suis meningitis. Front Microbiol 6:1001. https://doi.org/10.3389/fmicb.2015.01001

20. Xia X, Qin W, Zhu H, Wang X, Jiang J, Hu J (2019) How Streptococcus suis serotype 2 attempts to avoid attack by host immune defenses. J Microbiol Immunol 52:516-525. https://doi.org/10.1016/j.jmii.2019.03.003

21. Smith HE, Reek FH, Vecht U, Gielkens AL, Smits MA (1993) Repeats in an extracellular protein of weakly pathogenic strains of Streptococcus suis type 2 are absent in pathogenic strains. Infect Immun 61:3318-3326

22. Silva LM, Baums CG, Rehm T, Wisselink HJ, Goethe R, Valentin-Weigand P (2006) Virulence-associated gene profiling of Streptococcus suis isolates by PCR. Vet Microbiol 115:117-127. https://doi.org/10.1016/j.vetmi c.2005.12.013

23. Gottschalk MG, Lacouture S, Dubreuil JD (1995) Characterization of Streptococcus suis capsular type 2 haemolysin. Microbiology 141:189-195. https://doi.org/10.1099/00221287-141-1-189

24. Gottschalk M, Segura M (2000) The pathogenesis of the meningitis caused by Streptococcus suis: the unresolved questions. Vet Microbiol 76:259-272. https://doi.org/10.1016/s0378-1135(00)00250-9

25. Maiden MC, Jansen van Rensburg MJ, Bray JE, Earle SG, Ford SA, Jolley KA, McCarthy ND (2013) MLST revisited: the gene-by-gene approach to bacterial genomics. Nat Rev Microbiol 11:728-736. https://doi.org/10.1038/ nrmicro3093

26. King SJ, Leigh JA, Heath PJ, Luque I, Tarradas C, Dowson CG, Whatmore AM (2002) Development of a multilocus sequence typing scheme for the pig pathogen Streptococcus suis: identification of virulent clones and potential capsular serotype exchange. J Clin Microbiol 40:3671-3680. https://doi.org/10.1128/jcm.40.10.3671-3680.2002

27. Athey TB, Auger JP, Teatero S, Dumesnil A, Takamatsu D, Wasserscheid J, Dewar K, Gottschalk M, Fittipaldi N (2015) Complex population structure and virulence differences among serotype 2 Streptococcus suis strains belonging to sequence type 28. PLoS One 10:e0137760. https://doi. org/10.1371/journal.pone. 0137760

28. Segura M, Aragon V, Brockmeier SL, Gebhart C, Greeff A, Kerdsin A, O'Dea MA, Okura M, Saléry M, Schultsz C, Valentin-Weigand P, Weinert LA, Wells JM, Gottschalk M (2020) Update on Streptococcus suis research and prevention in the era of antimicrobial restriction: 4th International Workshop on S. suis. Pathogens 9:e374. https://doi.org/10.3390/patho gens9050374

29. Estrada AA, Gottschalk M, Rossow S, Rendahl A, Gebhart C, Marthaler DG (2019) Serotype and genotype (multilocus sequence type) of Streptococcus suis isolates from the United States serve as predictors of pathotype. J Clin Microbiol. https://doi.org/10.1128/jcm.00377-19

30. Rieckmann K, Pendzialek SM, Vahlenkamp T, Baums CG (2020) A critical review speculating on the protective efficacies of autogenous Streptococcus suis bacterins as used in Europe. Porcine Health Manag 6:12. https:// doi.org/10.1186/s40813-020-00150-6

31. Wileman TM, Weinert LA, Howell KJ, Wang J, Peters SE, Williamson SM, Wells JM, Langford PR, Rycroft AN, Wren BW, Maskell DJ, Tucker AW (2019) Pathotyping the zoonotic pathogen Streptococcus suis: novel genetic markers to differentiate invasive disease-associated isolates from nondisease-associated isolates from England and Wales. J Clin Microbiol. https://doi.org/10.1128/jcm.01712-18

32. Samanovic MI, Ding C, Thiele DJ, Darwin KH (2012) Copper in microbial pathogenesis: meddling with the metal. Cell Host Microbe 11:106-115. https://doi.org/10.1016/..chom.2012.01.009

33. Willemse N, Schultsz C (2016) Distribution of type I restriction-modifcation systems in Streptococcus suis: an outlook. Pathogens. https://doi. org/10.3390/pathogens5040062

34. Mitchell RN (2015) In: Kumar, V, Abbas, AK, Aster, JC, eds. Robbins and Cotran Pathologic Basis of Disease. Elsevier Saunders, Philadelphia, PA, p 1391

35. Sambrook J, Russel DW (2006) The condensed protocols from molecular cloning. In: A Laboratory Manual. Cold Spring Harbor Laboratory Press, New York

36. Ishida S, le Tien HT, Osawa R, Tohya M, Nomoto R, Kawamura Y, Takahashi T, Kikuchi N, Kikuchi K, Sekizaki T (2014) Development of an appropriate PCR system for the reclassification of Streptococcus suis. J Microbiol Meth 107:66-70. https://doi.org/10.1016/j.mimet.2014.09.003

37. Kerdsin A, Akeda Y, Hatrongjit R, Detchawna U, Sekizaki T, Hamada S, Gottschalk M, Oishi K (2014) Streptococcus suis serotyping by a new multiplex PCR. J Med Microbiol 63:824-830. https://doi.org/10.1099/ jmm.0.069757-0

38. Lane DJ (1991) 16S/23S rRNA sequencing. In: Stackebrandt E, Goodfellow $M$ (eds) Nucleic acid techniques in bacterial systematics. Wiley, New York, pp 115-175

39. Scherrer S, Rademacher F, Spoerry Serrano N, Schrenzel J, Gottschalk M, Stephan R, Landolt P (2020) Rapid high resolution melting assay to differentiate Streptococcus suis serotypes 2, 1/2, 1, and 14. Microbiol Open. https://doi.org/10.1002/mbo3.995

40. Rehm T, Baums CG, Strommenger B, Beyerbach M, Valentin-Weigand P, Goethe R (2007) Amplified fragment length polymorphism of Streptococcus suis strains correlates with their profile of virulence-associated genes and clinical background. J Med Microbiol 56:102-109. https://doi. org/10.1099/jmm.0.46616-0

41. Francisco AP, Bugalho M, Ramirez M, Carrico JA (2009) Global optimal eBURST analysis of multilocus typing data using a graphic matroid approach. BMC Bioinformatics 10:152. https://doi. org/10.1186/1471-2105-10-152

42. Zheng H, Ji S, Lan R, Liu Z, Bai X, Zhang W, Gottschalk M, Xu J (2014) Population analysis of Streptococcus suis isolates from slaughtered swine by use of minimum core genome sequence typing. J Clin Microbiol 52:3568-3572. https://doi.org/10.1128/jcm.00536-14

43. Stevens MJA, Spoerry Serrano N, Cernela N, Schmitt S, Schrenzel J, Stephan R (2019) Massive diversity in whole-genome sequences of Streptococcus suis strains from infected pigs in Switzerland. Microbiol Resour Announc. https://doi.org/10.1128/mra.01656-18

44. Prüfer TL, Rohde J, Verspohl J, Rohde M, de Greeff A, Willenborg J, Valentin-Weigand P (2019) Molecular typing of Streptococcus suis strains isolated from diseased and healthy pigs between 1996-2016. PLoS One 14:e0210801. https://doi.org/10.1371/journal.pone.0210801

45. Perch B, Pedersen KB, Henrichsen J (1983) Serology of capsulated streptococci pathogenic for pigs: six new serotypes of Streptococcus suis. J Clin Microbiol 17:993-996

46. Morales B, Ruiz A, Lacouture S, Gottschalk M (2015) Clonal distribution of Streptococcus suis isolated from diseased pigs in the central region of Chile. Can J Vet Res 79:343-346 
47. Dong W, Zhu Y, Ma Y, Ma J, Zhang Y, Yuan L, Pan Z, Wu Z, Yao H (2017) Multilocus sequence typing and virulence genotyping of Streptococcus suis serotype 9 isolates revealed high genetic and virulence diversity. FEMS Microbiol Lett. https://doi.org/10.1093/femsle/fnx192

48. Zheng H, Du P, Qiu X, Kerdsin A, Roy D, Bai X, Xu J, Vela Al, Gottschalk M (2018) Genomic comparisons of Streptococcus suis serotype 9 strains recovered from diseased pigs in Spain and Canada. Vet Res 49:1. https:// doi.org/10.1186/s13567-017-0498-2

49. Nathues $C$, Zimmerli U, Hauser R, Nathues $H$, Grosse Beilage $E$, Schüpbach-Regula G (2014) Risk assessment of the introduction of porcine reproductive and respiratory syndrome virus via boar semen into Switzerland as an example of a PRRSV-free country. Transbound Emerg Dis 61:546-554. https://doi.org/10.1111/tbed.12059

50. Stärk KD, Miserez R, Siegmann S, Ochs H, Infanger P, Schmidt J (2007) A successful national control programme for enzootic respiratory diseases in pigs in Switzerland. Rev Sci Tech 26:595-606

51. Wiederkehr DD, Sydler T, Buergi E, Haessig M, Zimmermann D, Pospischil A, Brugnera E, Sidler X (2009) A new emerging genotype subgroup within PCV-2b dominates the PMWS epizooty in Switzerland. Vet Microbiol 136:27-35. https://doi.org/10.1016/j.vetmic.2008.10.028
52. Olearo F, Marinosci A, Stephan R, Cherkaoui A, Renzi G, Gaia N, Leo S, Lazarevic V, Schrenzel J (2020) First case of Streptococcus suis infection in Switzerland: an emerging public health problem? Travel Med Infect Di. https://doi.org/10.1016/j.tmaid.2020.101590

53. Rieckmann K, Seydel A, Szewczyk K, Klimke K, Rungelrath V, Baums CG (2018) Streptococcus suis cps7: an emerging virulent sequence type (ST29) shows a distinct, IgM-determined pattern of bacterial survival in blood of piglets during the early adaptive immune response after weaning. Vet Res 49:48. https://doi.org/10.1186/s13567-018-0544-8

54. de Greeff A, Wisselink HJ, de Bree FM, Schultsz C, Baums CG, Thi HN, Stockhofe-Zurwieden N, Smith HE (2011) Genetic diversity of Streptococcus suis isolates as determined by comparative genome hybridization. BMC Microbiol 11:161. https://doi.org/10.1186/1471-2180-11-161

55. Yao X, Li M, Wang J, Wang C, Hu D, Zheng F, Pan X, Tan Y, Zhao Y, Hu L, Tang J, Hu F (2015) Isolation and characterization of a native avirulent strain of Streptococcus suis serotype 2: a perspective for vaccine development. Sci Rep 5:9835. https://doi.org/10.1038/srep09835

\section{Publisher's Note}

Springer Nature remains neutral with regard to jurisdictional claims in published maps and institutional affiliations.
Ready to submit your research? Choose BMC and benefit from:

- fast, convenient online submission

- thorough peer review by experienced researchers in your field

- rapid publication on acceptance

- support for research data, including large and complex data types

- gold Open Access which fosters wider collaboration and increased citations

- maximum visibility for your research: over 100M website views per year

At BMC, research is always in progress.

Learn more biomedcentral.com/submissions 